\title{
Structural transition in $\mathrm{Na}_{x} \mathrm{CoO}_{2}$ with $x$ near 0.75 due to $\mathrm{Na}$ rearrangement
}

\author{
Q. Huang, ${ }^{1}$ B. Khaykovich, ${ }^{2,3}$ F.C. Chou, ${ }^{2}$ J.H. Cho, ${ }^{3}$ J. W. Lynn, ${ }^{1}$ and Y.S. Lee ${ }^{2,3}$ \\ ${ }^{1}$ NIST Center for Neutron Research, NIST, Gaithersburg, MD 20899-8562 \\ ${ }^{2}$ Center for Materials Science and Engineering, Massachusetts Institute of Technology, Cambridge, MA 02139 \\ ${ }^{3}$ Department of Physics, Massachusetts Institute of Technology, Cambridge, MA 02139
}

(Dated: July 3, 2018)

\begin{abstract}
We report neutron powder diffraction measurements on a series of $\mathrm{Na}_{x} \mathrm{CoO}_{2}$ samples with $x$ near 0.75 which were prepared under different synthesis conditions. Two different crystal structures for the samples are observed at room temperature. The two structures differ primarily by a shift of a large fraction of the $\mathrm{Na}$ ions from a high-symmetry position to a lower-symmetry position. Close inspection of the refinement parameters indicates that the presence of either structure depends sensitively on the Na content $x$, with $x \simeq 0.75$ as the critical concentration separating the two phases. By raising the temperature to around $T \simeq 323 \mathrm{~K}$, the high-symmetry structure can be converted to the lower-symmetry structure. The transition is reversible, but there is significant hysteresis. We discuss the effects of this structural transition on the bulk magnetic and transport properties.

PACS numbers: 74.72.Dn, 74.40.+k, 75.30.Fv, 75.10.Jm, 75.50.Ee
\end{abstract}

\section{INTRODUCTION}

The layered cobaltate $\mathrm{Na}_{x} \mathrm{CoO}_{2}$ has generated much recent interest as a correlated electron material with unusual electronic properties. The structure of this compound consists of layers of Co atoms, within an octahedral environment of oxygen atoms, arranged on a triangular lattice. The $\mathrm{Na}$ atoms form layers in between the $\mathrm{CoO}_{2}$ layers, and the $\mathrm{Na}$ stoichiometry can vary from $x \sim 0.25$ to 0.85 . This family of materials has attracted much recent interest due to the discovery of superconductivity below $4.5 \mathrm{~K}$ in hydrated $\mathrm{Na}_{0.3} \mathrm{CoO}_{2}$ $\cdot 1.3 \mathrm{H}_{2} \mathrm{O} .1$ The composition with $x \approx 0.75$ shows an unusually strong thermoelectric effect and has Curie-Weiss magnetism coexisting with metallic behavior ${ }^{2.3}$ The electronic phase diagram of non-hydrated $\mathrm{Na}_{x} \mathrm{CoO}_{2}$ is rich, with two metallic phases existing for $x>0.5$ and $x<0.5$ which are separated by a charge-ordered insulator at $x=0.5^{4.5}$ It is clear that the properties of $\mathrm{Na}_{x} \mathrm{CoO}_{2}$ can be strongly affected by the mobility of the $\mathrm{Na}$ ions, which leads, for example, to the formation of a $\mathrm{Na}$ superlattice at $x=0.5^{4.6}$ In order to further understand the electronic and magnetic properties of $\mathrm{Na}_{x} \mathrm{CoO}_{2}$, it is helpful to first understand the details of the crystal structure.

In this paper we present neutron powder diffraction measurements of samples of $\mathrm{Na}_{x} \mathrm{CoO}_{2}$ with $x$ near 0.75 . We find that there exist two distinct structures in this region of the phase diagram. These structures differ from each other by the arrangement of the $\mathrm{Na}$ ions. In addition, we find that the $\mathrm{Na}$ arrangement depends sensitively on the temperature and doping level $x$. These results are important because they demonstrate that samples of nominally the same composition, $x \sim 0.75$, may have different physical properties. Recently, Sales and coworkers have found evidence for a transition around $340 \mathrm{~K}$ using scanning calorimetry, magnetization and transport measurements $?^{7}$ They attributed this behavior to a pos- sible structural transition involving $\mathrm{Na}$ ordering. Our powder neutron diffraction measurements directly reveal how the crystal structure changes. We present results from a systematic study involving neutron diffraction, resistivity measurements, and susceptibility measurements on both single crystal and powder samples annealed under various conditions.

\section{EXPERIMENTAL}

Powder samples of $\mathrm{Na}_{x} \mathrm{CoO}_{2}$ were prepared using a solid-state reaction technique. A mixture of $0.75 \mathrm{Na}_{2} \mathrm{CO}_{3}$ $+2 / 3 \mathrm{Co}_{3} \mathrm{O}_{4}$ was used as the starting material. The material was reacted at elevated temperatures $\left(750^{\circ} \mathrm{C}\right.$ and $900^{\circ} \mathrm{C}$ ) in air with repeated grindings over 12 hours cycles until single phase $\mathrm{Na}_{0.75} \mathrm{CoO}_{2}$ was achieved, as verified with $\mathrm{x}$-ray diffraction. The single crystal samples were grown via the travelling solvent floating-zone method as described previously. ${ }^{8}$ In this paper, we have examined the structure of four different samples: (i) a crushed single crystal labelled CC, (ii) an as-prepared powder which was quenched in air from $900^{\circ} \mathrm{C}$ labelled P-900, (iii) an as-prepared powder quenched in air from $750^{\circ} \mathrm{C}$ labelled P-750, and (iv) a powder sample labelled $\mathbf{P}$-anneal which was annealed in a flowing oxygen atmosphere while slowly cooled between from $900^{\circ}$ to $50^{\circ} \mathrm{C}$ at a rate of $-1^{\circ} \mathrm{C} / \mathrm{min}$. The samples' histories are summarized in Table II All four samples originated from different batches and therefore have slight variations in stoichiometry $x$ near 0.75 .

The neutron powder diffraction data of these samples were collected using the BT-1 high-resolution powder diffractometer at the NIST Center for Neutron Research, employing a $\mathrm{Cu}$ (311) monochromator crystal to produce a beam of monochromatic neutrons with wavelength $\lambda=1.5403 \AA$. Collimators with horizontal angular divergences of $15^{\prime}, 20^{\prime}$, and $7^{\prime}$ were used before and after 
TABLE I: Summary of $\mathrm{Na}_{0.75} \mathrm{CoO}_{2}$ samples

\begin{tabular}{ccccc}
\hline \hline name & batch\# & sample form & reaction atm. & highest reaction temp. \\
\hline CC & $\# 2465$ & crushed crystal & oxygen & $>1100^{\circ} \mathrm{C}$ \\
P-900 & $\# 2683$ & powder & air & $900^{\circ} \mathrm{C}$ \\
P-750 & $\# 2683 B$ & powder & air & $750^{\circ} \mathrm{C}$ \\
P-anneal & $\# 2683 D$ & powder & air $+\mathrm{O}_{2}$ annealed & $900^{\circ} \mathrm{C}$ \\
\hline \hline
\end{tabular}

the monochromator, and after the sample, respectively. The scattered intensities were measured as a function of $2 \theta$ in steps of $0.05^{\circ}$ in the range $3^{\circ}-168^{\circ}$. The structural parameters were refined using the GSAS program 9 The neutron scattering lengths used in the refinements were $0.363,0.253$, and $0.581\left(\times 10^{-12} \mathrm{~cm}\right)$ for $\mathrm{Na}, \mathrm{Co}$, and $\mathrm{O}$, respectively.

Magnetization measurements were performed using a SQUID magnetometer (Quantum Design MPMS-XL). The resistivity of a single crystal sample was measured using the standard 4-probe technique. Electrical contacts were attached to the surface of the sample with silver epoxy and measurements were performed from $5 \mathrm{~K}$ to $350 \mathrm{~K}$ in applied fields of 0 Tesla and 14 Tesla using a Physical Property Measurement System (Quantum Design).

\section{RESULTS}

We first present our results on the structural properties of our samples which all have the same nominal $\mathrm{Na}$ concentration near $x=0.75$. The structure of $\mathrm{Na}_{x} \mathrm{CoO}_{2}$ consists of layers of edge-sharing $\mathrm{CoO}_{6}$ octahedra, where the Co atoms form a triangular lattice. For the hexagonal unit cell, the lattice constants are $a_{H} \simeq 2.84$ and $c_{H} \simeq 10.82 \AA$. The Na ions occupy planes which lie in between the $\mathrm{CoO}_{2}$ layers. Previous measurements ${ }^{10,11}$ have revealed two crystallographically distinct sites which the $\mathrm{Na}$ ions occupy in the lattice, $\mathrm{Na}(1)$ and $\mathrm{Na}(2)$. The relative population of these sites varies from sample to sample and depends strongly on the Na concentration $x$.

Figure 1 shows a portion of the neutron powder diffraction pattern of $\mathrm{Na}_{0.75} \mathrm{CoO}_{2}$ for the crushed single crystal sample (CC) and two of the powder samples (P-900 and $\mathbf{P - 7 5 0}$ ). By comparing the room-temperature pattern for sample $\mathbf{C C}$ (top-most profile) with the pattern for sample P-750 (bottom-most profile), we find that there are two distinct structural phases. As indicated in the plot, the positions of several peaks (such as the (106) and (107) peaks) are clearly different, indicating slight differences in the crystal structure. The data can be fit using the hexagonal space group $P 6_{3} / m m c$ with structural models $\mathrm{H} 1$ or $\mathrm{H} 2$ as shown in Fig. 2.10 .11 These structural models will be discussed in detail below. The primary difference is that the $\mathrm{Na}(2)$ ions reside at a higher symmetry position for structure $\mathrm{H} 2$ compared to structure H1. The crushed crystal CC has structure H2 at room temperature, whereas the powder sample $\mathbf{P}-\mathbf{7 5 0}$

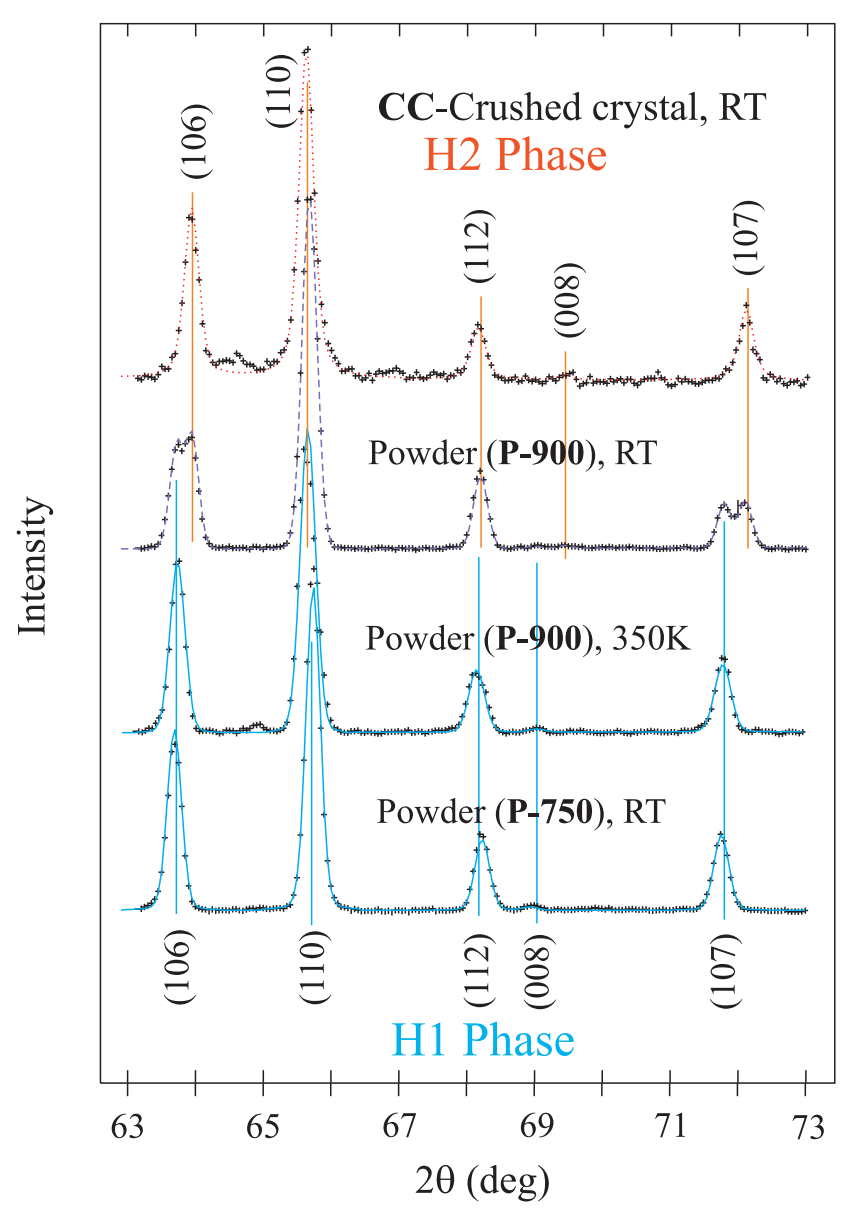

FIG. 1: (color online) Portions of the neutron powder diffraction pattern of $\mathrm{Na}_{x} \mathrm{CoO}_{2}$ samples annealed under different conditions. The graphs from top to bottom correspond to the following samples: crushed crystal CC measured at room temperature (RT); powder P-900 quenched in air from $900^{\circ} \mathrm{C}$ measured at RT; powder P-900 measured at $350 \mathrm{~K}$; powder P-750 quenched in air from $750^{\circ} \mathrm{C}$ measured at RT. The curves are the calculated intensity with the structural models H2 (red-dotted) and H1 (blue-solid), corresponding to the crystal structures shown on Fig. 2

has structure $\mathrm{H} 1$ at room temperature.

The middle two diffraction profiles in Fig. 1 were taken on sample $\mathbf{P - 9 0 0}$ at room temperature and at $T=350 \mathrm{~K}$. Interestingly, the room temperature pattern shows coexistence of both structures, H1 and H2. When this sample is heated to $350 \mathrm{~K}$, the peaks associated with structure H2 disappear completely, and the peaks associated with 


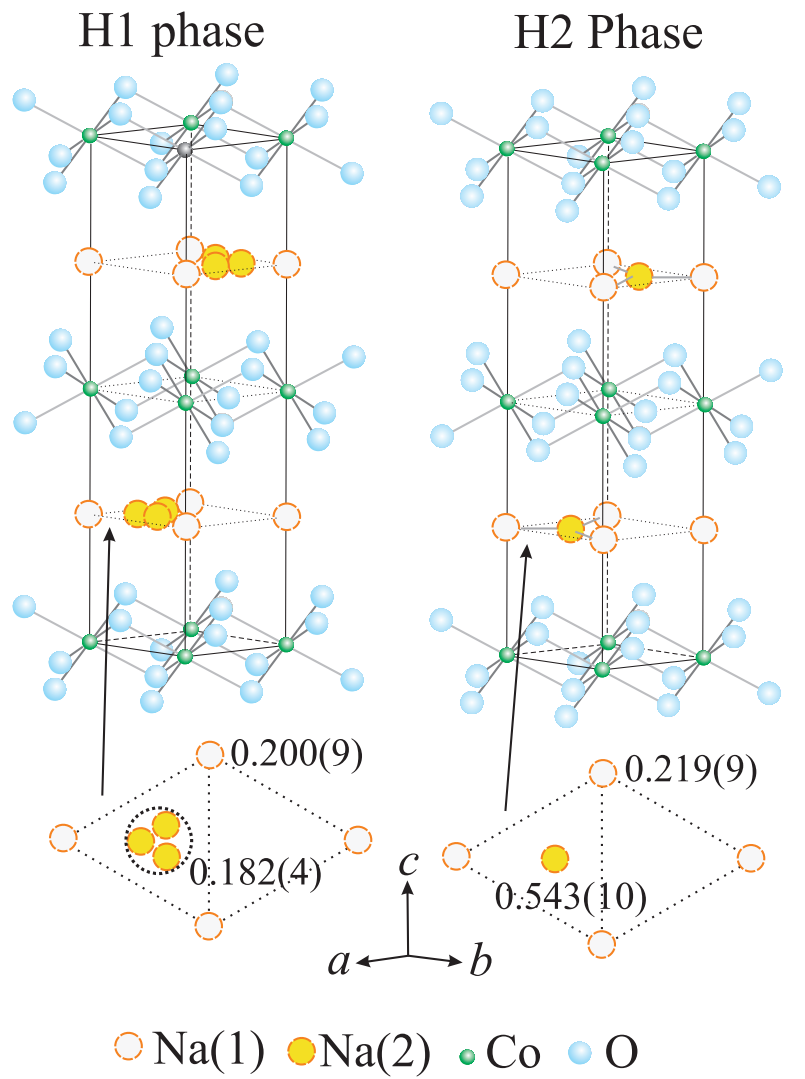

(a)

FIG. 2: (color online) Models for the crystal structure used in the refinements for $\mathrm{Na}_{x} \mathrm{CoO}_{2}$ ( $x$ near 0.75 ), space group $P 6_{3} / m m c$. The $\mathrm{Na}(2)$ atoms are (a) at the $6 h(2 x, x, 1 / 4)$ site for structure $\mathrm{H} 1$ and (b) at the $2 c(2 / 3,1 / 3,1 / 4)$ site for structure H2. The numbers shown in the lower figure of the $\mathrm{Na}$ planes are the refined occupancy of each $\mathrm{Na}$ site for the P-900 sample.

structure H1 are enhanced. This indicates that a structural phase transition occurs at an intermediate temperature. Note that the powder profile of the $\mathbf{P - 9 0 0}$ sample at $350 \mathrm{~K}$ is identical to the profile for the $\mathbf{P} \mathbf{- 7 5 0}$ sample at room temperature, demonstrating that the conversion of the phase with structure $\mathrm{H} 2$ to the phase with structure $\mathrm{H} 1$ is complete. The diffraction pattern for the sample $\mathbf{P}$-anneal, which was slow-cooled in oxygen, shows that the entire sample is described by structure H1. The structural parameters for all four samples determined by Rietveld refinement are summarized in Table III

In order to characterize the structural phase transition in more detail, we measured the (109) Bragg peak of sample P-900 over a range of temperatures. As shown in Figure 3(a), at a temperature of $T=290 \mathrm{~K}$ there are two diffraction peaks, (109)-H1 and (109)-H2, signifying the coexistence of both structures ( $\mathrm{H} 1$ and $\mathrm{H} 2)$. As the temperature is raised in steps of $2 \mathrm{~K}$, the intensity of the (109)-H2 peak decreases and the intensity of (109)-H1
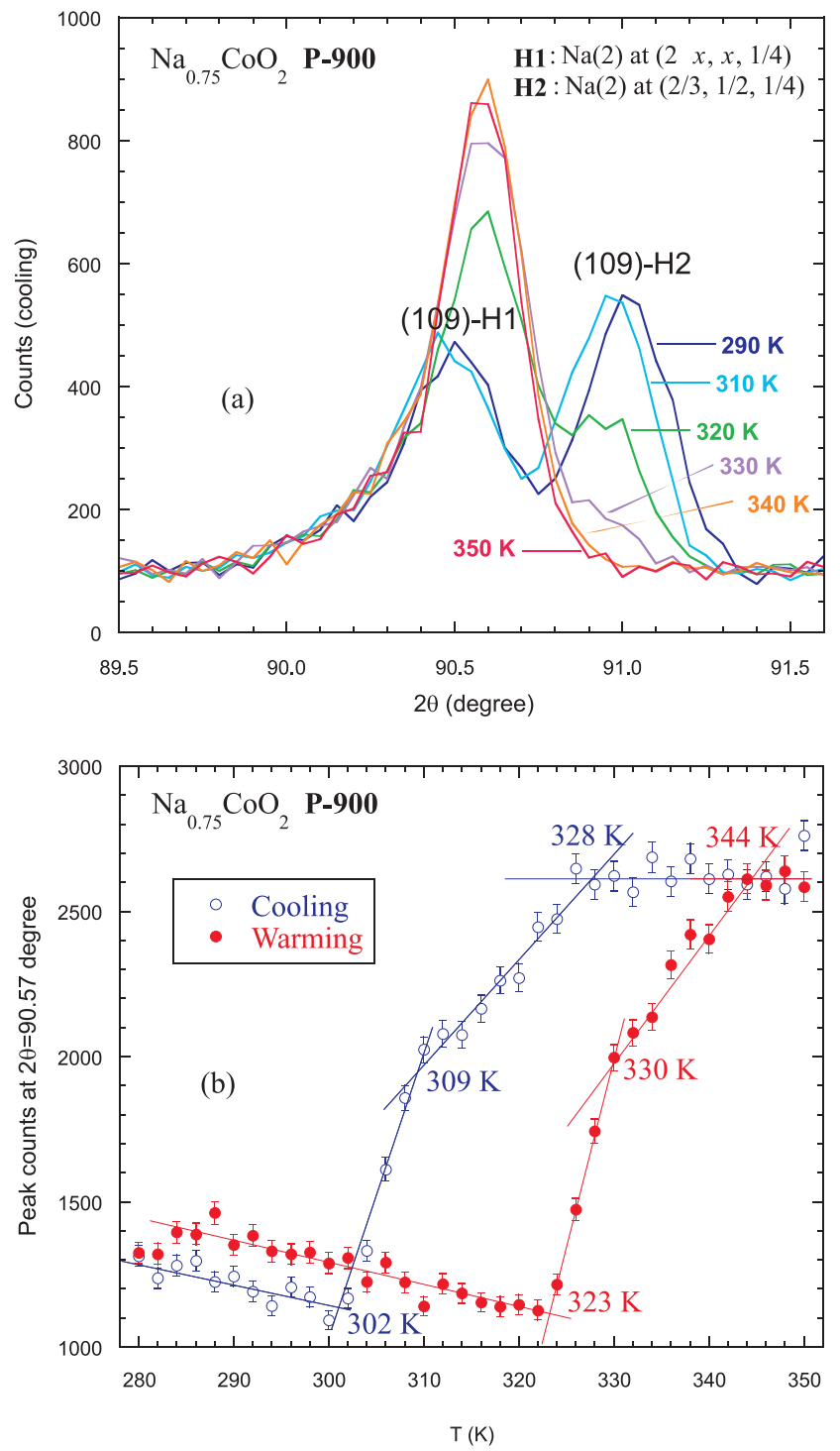

FIG. 3: (color online) Top panel: scans through the the (109) peak of sample P-900 at various temperatures. Bottom panel: temperature dependence of the intensity of the (109)H1 peak. These data demonstrate that at high temperatures (above $340 \mathrm{~K}$ ), model $\mathrm{H} 1$ is the stable structure.

peak increases. Upon warming, the sample becomes single phase above $340 \mathrm{~K}$, possessing only the H1 structure. Figure 3(b) shows the intensity at a $2 \theta$-position corresponding to the (109)-H1 reflection as a function of temperature. The intensity suddenly increases above $323 \mathrm{~K}$ upon warming, and reaches a maximum near $344 \mathrm{~K}$. Upon cooling the intensity begins to decrease suddenly below $328 \mathrm{~K}$ and levels off below $302 \mathrm{~K}$. The marked hysteresis (around 20 degrees in temperature) suggests that the transformation from structure $\mathrm{H} 2$ to $\mathrm{H} 1$ is a first order transition.

The reason for the coexistence of the two structures at room temperature is that sample $\mathbf{P - 9 0 0}$ is likely composed of a mixture of two stoichiometries with slightly 
TABLE II: Structural parameters for $\mathrm{Na}_{x} \mathrm{CoO}_{2}(x \simeq 0.75)$. Space group $P 6_{3} / m m c$. Atomic positions: Co: $2 \mathrm{a}(0$ 0 0); Na(1): $2 \mathrm{~b}(001 / 4) ; \mathrm{Na}(2)$ : $2 \mathrm{c}(2 / 3,1 / 3,1 / 4)$ for structure model A or $6 \mathrm{~h}(2 \mathrm{x}, \mathrm{x}, 1 / 4)$ for structure model $\mathrm{B} ; \mathrm{O}: 4 \mathrm{f}(1 / 3,2 / 3, \mathrm{z})$.

\begin{tabular}{|c|c|c|c|c|c|c|c|c|c|c|c|c|c|c|c|}
\hline \multirow{3}{*}{$\begin{array}{l}\text { Sample ID } \\
\mathrm{T}(\mathrm{K}) \\
x \text { (refined) }\end{array}$} & & \multicolumn{5}{|c|}{$P-750$} & \multicolumn{7}{|c|}{ P-900 ( $3.6 \mathrm{wt} \% \mathrm{CoO})$} & \multirow{3}{*}{\begin{tabular}{|l|} 
P-anneal \\
295 \\
$0.749(8)$
\end{tabular}} & \multirow{3}{*}{\begin{tabular}{l}
\multicolumn{1}{c|}{$\mathbf{C C}$} \\
295 \\
$0.752(17)$
\end{tabular}} \\
\hline & & \multirow{2}{*}{\multicolumn{2}{|c|}{$\begin{array}{l}3.6 \\
0.721(8)\end{array}$}} & \multirow{2}{*}{\multicolumn{2}{|c|}{$\begin{array}{l}30 \\
0.726(8)\end{array}$}} & \multirow{2}{*}{$\begin{array}{l}295 \\
0.711(8)\end{array}$} & \multicolumn{4}{|c|}{295} & \multicolumn{3}{|c|}{350} & & \\
\hline & & & & & & & \multicolumn{2}{|l|}{$0.746(15)$} & \multicolumn{2}{|c|}{$0.762(13)$} & \multicolumn{2}{|c|}{0.746} & 0.762 & & \\
\hline \multicolumn{2}{|l|}{$\begin{array}{l}\text { Structure } \\
\mathrm{a}(\AA)\end{array}$} & \multicolumn{2}{|l|}{ H1 } & \multicolumn{2}{|c|}{ H1 } & H1 & \multicolumn{2}{|c|}{ H1(46.2(2)\%) } & \multicolumn{2}{|c|}{ H2(50.2(2)\%) } & \multicolumn{2}{|c|}{ H1 $(42.9(7) \%)$} & H1 $(53.4(6) \%)$ & H1 & H2 \\
\hline $\mathrm{a}(\AA)$ & & \multicolumn{2}{|c|}{$2.83607(4)$} & \multicolumn{2}{|c|}{$2.83603(4)$} & $2.83709(4)$ & \multicolumn{2}{|l|}{$2.83709(5)$} & \multicolumn{2}{|c|}{$2.84064(4)$} & \multicolumn{2}{|c|}{$2.8369(1)$} & $2.84250(9)$ & $2.83628(5)$ & $2.84081(9)$ \\
\hline $\mathrm{c}(\AA)$ & & 10.8 & $25(2)$ & 10.8327 & $7(2)$ & $10.8811(2)$ & $10.8735(2)$ & & 10.8117 & & 10.87 & $33(5)$ & $10.8611(5)$ & $10.8863(3)$ & $10.8115(5)$ \\
\hline $\mathrm{V}\left(\AA^{3}\right)$ & & 75.45 & (3) & 75.455 & & $75.849(3)$ & $75.796(3)$ & & 75.5541 & & 75.78 & $5(5)$ & $75.998(5)$ & $75.842(4)$ & $75.562(6)$ \\
\hline Co & $\mathrm{B}\left(\AA^{2}\right)$ & 0.13 & & $0.14(2)$ & & $0.32(2)$ & $0.20(3)$ & & $0.20(3)$ & & 0.20 & & $0.20(3)$ & $0.38(4)$ & $0.13(7)$ \\
\hline $\mathrm{Na}(1)$ & $\mathrm{B}\left(\AA^{2}\right)$ & 0.31 & & $0.47(8)$ & & $1.04(9)$ & $1.30(8)$ & & $1.30(8)$ & & 1.41( & & $1.41(9)$ & $1.7(1)$ & $1.0(1)$ \\
\hline & $n$ & 0.196 & & $0.198(5$ & & $0.198(5)$ & $0.200(9)$ & & $0.219(9$ & & 0.200 & & 0.219 & $0.221(5)$ & $0.224(11)$ \\
\hline $\mathrm{Na}(2)$ & $x$ & 0.281 & $(7)$ & 0.2814( & & $0.2834(8)$ & $0.274(1)$ & & & & 0.285 & & $0.299(2)$ & $0.289(2)$ & \\
\hline & $\mathrm{B}\left(\AA^{2}\right)$ & 0.31( & & $0.47(8)$ & & $1.04(9)$ & $1.30(8)$ & & $1.30(8)$ & & 1.41( & & $1.41(9)$ & $1.7(1)$ & \\
\hline & $\mathrm{n}$ & 0.175 & & $0.176(2$ & & $0.171(2)$ & $0.182(4)$ & & $0.543(1$ & & 0.182 & & 0.181 & $0.176(2)$ & $0.518(13)$ \\
\hline $\mathrm{O}$ & $z$ & 0.090 & $4(7)$ & 0.09052 & $2(6)$ & $0.09023(7)$ & ) $0.0904(1)$ & & 0.0909 ( & & 0.092 & $5(2)$ & $0.0888(2)$ & $0.08990(8)$ & $0.0908(2)$ \\
\hline & $\mathrm{B}\left(\AA^{2}\right)$ & 0.43 & & $0.45(1)$ & & $0.61(1)$ & $0.46(1)$ & & $0.46(1)$ & & 0.60 & & $0.60(2)$ & $0.56(2)$ & $0.50(3)$ \\
\hline & $R_{p}(\%)$ & 4.75 & & 4.62 & & 4.42 & & 4.1 & & & & 5. & 28 & 4.90 & 4.54 \\
\hline & $R_{w p}$ & 5.90 & & 5.78 & & 5.39 & & 5.0 & & & & 6. & 58 & 6.54 & 5.66 \\
\hline & $\chi^{2}$ & 2.100 & & 1.976 & & 1.790 & & 1.6 & & & & 2.1 & 149 & 2.829 & 1.323 \\
\hline & & & & & & Selecte & d bond $(\AA)$ & distan & aces and & angles & (degre & & & & \\
\hline $\mathrm{Co}-\mathrm{O}$ & & $\times 6$ & 1.908 & $87(4)$ & 1.9 & $085(4)$ & $1.9097(4)$ & 1.91 & $03(6)$ & 1.9115 & $9(6)$ & $1.922(1)$ & $1.904(1)$ & $1.9077(5)$ & $1.912(1)$ \\
\hline O-Co-O & & & 95.97 & $7(3)$ & & $97(3)$ & $95.94(2)$ & 95.9 & $0(4)$ & 95.961 & & $95.11(9)$ & $96.59(8)$ & $96.04(3)$ & $95.97(7)$ \\
\hline $\mathrm{Na}(1)-\mathrm{O}$ & & $\times 6$ & 2.380 & $01(5)$ & 2.3 & $803(5)$ & $2.3886(5)$ & 2.38 & $363(8)$ & 2.3768 & $8(8)$ & $2.370(2)$ & $2.399(2)$ & $2.3915(6)$ & $2.377(2)$ \\
\hline $\mathrm{Na}(2)-\mathrm{O}$ & & $\times 4$ & 2.304 & 4(1) & 2.3 & $05(1)$ & $2.316(1)$ & 2.30 & $2(2)$ & 2.3768 & $8(8)$ & $2.298(3)$ & $2.346(4)$ & $2.326(2)$ & $2.377(2)$ \\
\hline & & $\times 2$ & 2.564 & 4(3) & 2.5 & $62(3)$ & $2.563(3)$ & 2.59 & $6(5)$ & 2.3768 & $8(8)$ & $2.540(8)$ & $2.519(9)$ & $2.547(5)$ & $2.377(2)$ \\
\hline $\mathrm{Na}(2)-\mathrm{O}_{a v}$ & rage & & 2.391 & $1(5)$ & 2.3 & $89(5)$ & $2.398(5)$ & 2.40 & $0(8)$ & 2.3768 & $8(8)$ & $2.38(1)$ & $2.40(2)$ & $2.400(8)$ & $2.377(2)$ \\
\hline
\end{tabular}

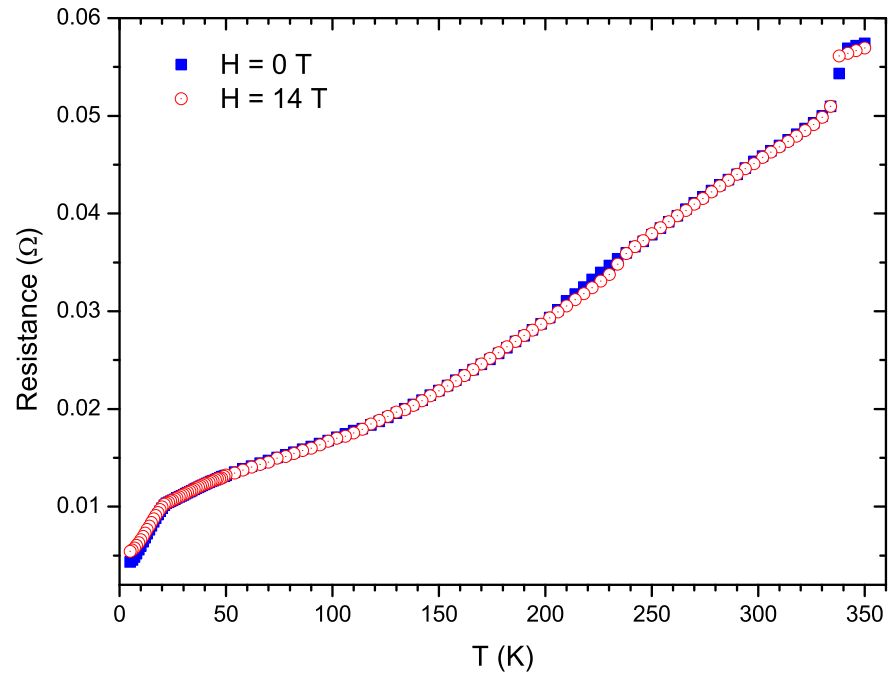

FIG. 4: (color online) Resistivity versus temperature on a single crystal prepared under the same conditions as the crushed crystal sample CC.

different $\mathrm{Na}$ contents. The Rietveld refinement at $T=$ $295 \mathrm{~K}$ indicates the sample is almost an equal mixture of two compounds with $x=0.746(15)$ and $x=0.762(13)$. (We note that the error bars for $x$ for these two phases overlap. Alternatively, we could label these two phases

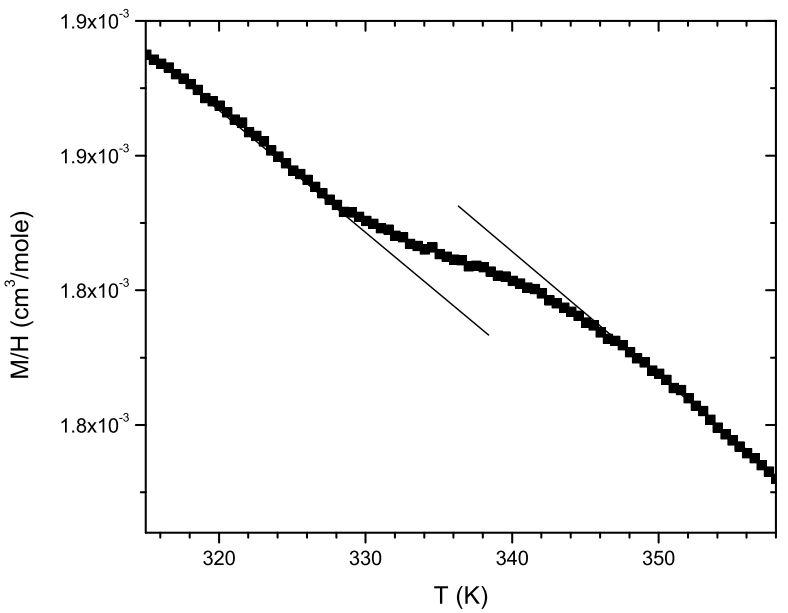

FIG. 5: (color online) Magnetic susceptibility versus temperature on the same sample as on Fig. 4 A magnetic field of 5 $\mathrm{T}$ was applied approximately parallel to the $a b$-plane.

using their $a$ and $c$ lattice constants, which are clearly distinguishable.) A complete diffraction pattern was collected at $350 \mathrm{~K}$ and the data were analyzed by the Rietveld method using a two-phase model with the same 
H1 structure but different structural parameters. In this refinement the Na content $x$ and the occupancies for each site were fixed at values obtained from the $295 \mathrm{~K}$ data, assuming that the $\mathrm{Na}$ atoms do not change sites (ie. $\mathrm{Na}(1)$ and $\mathrm{Na}(2)$ ) between 295 and $350 \mathrm{~K}$. The results are shown in Table III From these fits, we can conclude that the temperature dependence plotted in Fig. 3 is entirely derived from the phase associated with $x=0.762$.

We have measured the transport properties of a single crystal sample of $\mathrm{Na}_{0.75} \mathrm{CoO}_{2}$ which was prepared under the same conditions as sample CC. Figure 4 shows the resistance as a function of temperature between $5 \mathrm{~K}$ and $350 \mathrm{~K}$, measured in magnetic fields of 0 Tesla and 14 Tesla oriented along the c-axis. The most noticeable features of the resistivity curves are the transitions near $22 \mathrm{~K}$ and $340 \mathrm{~K}$. Both of these features agree with the reported measurements of Sales et.al. on a floating-zone grown $\mathrm{Na}_{0.75} \mathrm{CoO}_{2}$ sample $\frac{7}{\underline{7}}$ The distinct jump in resistivity between $330 \mathrm{~K}$ and $340 \mathrm{~K}$ (upon warming) in Fig. प closely matches the temperature range (between $323 \mathrm{~K}$ and 344 $\mathrm{K})$ over which the H2 structure becomes transformed to the H1 structure for the sample shown in Fig. 3 Since the crushed crystal is also described by the $\mathrm{H} 2$ structure at room temperature, we conclude that the jump in resistance near $340 \mathrm{~K}$ is a direct result of the structural transition $(\mathrm{H} 2 \rightarrow \mathrm{H} 1)$ that we have discovered. We also observe a very small magnetoresistance effect between $200 \mathrm{~K}$ and $240 \mathrm{~K}$.

Magnetic susceptibility measurements were performed on the same single crystal used for the resistance measurement. These data are shown in Fig. 5 We find that there is a subtle change in the temperature dependence between $330 \mathrm{~K}$ and $340 \mathrm{~K}$. This is consistent with other reported measurements $\underline{\underline{7}}$ Again, we can associate this behavior with the newly discovered structural transition. Measurements of the magnetic susceptibility of the crushed crystal sample $\mathbf{C C}$ show nearly identical behavior.

\section{DISCUSSION}

The most important finding of this work is the existence of two distinct structures for $\mathrm{Na}_{x} \mathrm{CoO}_{2}$ samples with $x$ near 0.75 . The difference between the two structural models, $\mathrm{H} 1$ and $\mathrm{H} 2$, is that in model $\mathrm{H} 2$ the $\mathrm{Na}(2)$ atoms occupy the $2 c(2 / 3,1 / 3,1 / 4)$ site, while for the model $\mathrm{H} 1$ structure the $\mathrm{Na}(2)$ atoms randomly occupy the $6 h(2 x, x, 1 / 4)$ site. To emphasize the differences in $\mathrm{Na}$ positions in the two models, we show a section of a $\mathrm{Na}$ layer, projected along the c-axis, in the lower part of the Fig. 2] In addition, Fig. [6] shows the coordination of the $\mathrm{Na}(1)$ and $\mathrm{Na}(2)$ sites. There are six oxygen atoms surrounding the $\mathrm{Na}$ with the average $\mathrm{Na}-\mathrm{O}$ bond distance $\sim 2.38 \AA$ A, forming a triangular polyhedron. The $\mathrm{Na}(1) \mathrm{O}_{6}$ triangular polyhedron is face sharing with two $\mathrm{CoO}_{6}$ octahedra (directly above and below) and the $\mathrm{Na}(2) \mathrm{O}_{6}$ triangular polyhedron is corner sharing with six $\mathrm{CoO}_{6}$ octa-

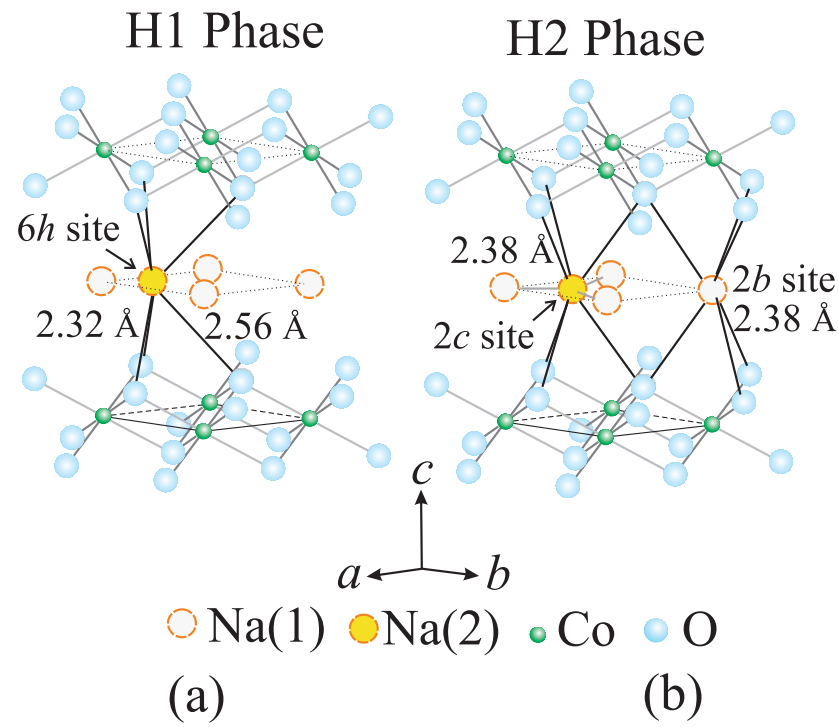

FIG. 6: (color online) Coordination of $\mathrm{Na}(1)$ and $\mathrm{Na}(2)$ in models $\mathrm{H} 1$ and $\mathrm{H} 2$. The $\mathrm{Na}(1)$ ions are located at the $2 b$ site, center of $\mathrm{NaO}_{6}$ triangular polyhedron, and are coordinated to six oxygen atoms which are associated with two nearest $\mathrm{CoO}_{6}$ octahedra (directly above and below). (a) The $\mathrm{Na}(2)$ ions randomly occupy the $6 \mathrm{~h}$ site, shifting away from the $2 \mathrm{c}$ site, i.e. the center of the triangular polyhedron. (b) The $\mathrm{Na}(2)$ ions are located at the $2 \mathrm{c}$ site, the center of the triangular mesh, and are coordinated to six oxygen atoms which are associated with to six nearest $\mathrm{CoO}_{6}$ octahedra (above and below $\mathrm{CoO}_{2}$ blocks).

hedra in the nearest $\mathrm{CoO}_{2}$ blocks. Both $\mathrm{Na}(1)$ and $\mathrm{Na}(2)$ sites are partially occupied and the ratio of the number of the $\mathrm{Na}$ ions is $\mathrm{n}_{N a(2)}: \mathrm{n}_{N a(1)}=2.5: 1$. No evidence of vacancy ordering was observed in our experiments. In model $\mathrm{H} 2$, the $\mathrm{Na}(1)$ and $\mathrm{Na}(2)$ ions are located at the corner and center of the triangular mesh of the hexagonal lattice, respectively, and both $\mathrm{Na}(1)$ and $\mathrm{Na}(2)$ have identical bonding distances to the nearest oxygen atoms. In contrast, in model $\mathrm{H} 1$, the $\mathrm{Na}(2)$ shifts away from the center of the Co-ions triangle and randomly occupies the $6 \mathrm{~h}(2 \mathrm{x}, \mathrm{x}, 1 / 4)$ sites. In this case, therefore, the $\mathrm{Na}$ ions shift away from the center of the triangular $\mathrm{NaO}_{6}$ polyhedron resulting in two long $(2.56 \AA)$ and four short $(2.32 \AA) \mathrm{Na}-\mathrm{O}$ bonds.

Another important result from our work is shown in Fig. 7 Here, we plot of the lattice constants as a function of Na content $x$, where $x$ was determined by Rietveld refinement as listed in TableII In samples with structure $\mathrm{H} 1$, the a-axis is significantly shorter and the c-axis is significantly longer than in samples with structure $\mathrm{H} 2$. Hence, these two structures can be readily distinguished from each other. Our refinements suggest that a sudden change occurs at $x$ near 0.75 , in which structure $\mathrm{H} 1$ is the stable phase below $x \simeq 0.75$ and structure $\mathrm{H} 2$ is the stable phase above $x \simeq 0.75$. We note that the error bars for the refined values of $x$ are large and overlap, except for the two samples: P-750 with $x=0.711$ and 


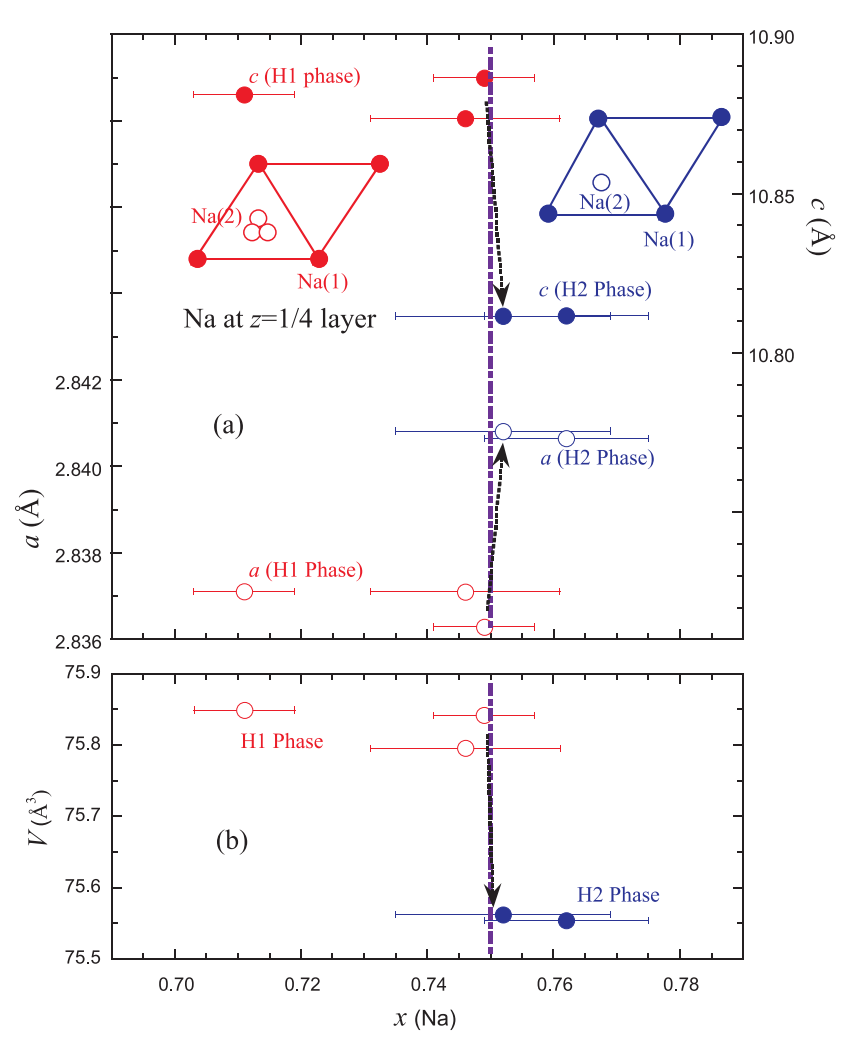

FIG. 7: (color online) Lattice parameters at room temperature as a function of $\mathrm{Na}$ content $x$. The change in the lattice constants as $x$ increases above $x \simeq 0.75$ corresponds to the change from the $\mathrm{H} 1$ structure to the $\mathrm{H} 2$ structure.

P-900 with $x=0.762$. However, the identification of $x=0.75$ as the critical concentration is further confirmed by studies on other samples of $\mathrm{Na}_{x} \mathrm{CoO}_{2}$ with different stoichiometry $(0.3<x<1) .12$ We note that the H1to-H2 phase transition is not observed in sample $\mathbf{P}-\mathbf{7 5 0}$ which has $x=0.711$. At this value of $x$, the structure $\mathrm{H} 1$ is stable for the entire temperature range measured.

The structural phase transition resembles an orderdisorder phase transitions in that $\mathrm{Na}$ atoms shift from a random occupation of the $6 h$ sites (structure H1) to a configuration in which every $\mathrm{Na}(2)$ atom occupies a $2 c$ position (structure $\mathrm{H} 2$ ). The transition is unusual in that it is the lower temperature phase (structure H2) which has the higher symmetry for the $\mathrm{Na}(2)$ ions. How- ever, we note that both structures have the same average space group symmetry $P 6_{3} / m m c$, as measured by neutron powder diffraction.

The sudden expansion of the $c$-axis lattice constant at the transition from structure $\mathrm{H} 1$ to $\mathrm{H} 2$ may explain the sharp jump in the resistance that we measured near $340 \mathrm{~K}$ upon warming. The transport properties of $\mathrm{Na}_{x} \mathrm{CoO}_{2}$ are highly anisotropic, with the resistivity within the $a b$-planes being several hundred times higher than that perpendicular to the planes ${ }^{7}$ The resistance of such an anisotropic material measured using the 4-probe technique usually contains an out-of-plane component due to non-uniform current flow across the sample. Since the caxis resistivity should increase for a larger c-axis lattice constant, this may explain the jump in our resistance data shown in Fig. 4 Alternatively, the jump in resistivity may be related to enhanced scattering due to the disorder in the $\mathrm{H} 1$ structure, which has randomly occupied $6 h(2 x, x, 1 / 4)$ sites.

In conclusion, we have found two distinct structural phases in $\mathrm{Na}_{x} \mathrm{CoO}_{2}$ with $x$ near 0.75 . These two phase are characterized by $\mathrm{Na}$ ions which occupy different sites, $6 h(2 x, x, 1 / 4)$ in structure $\mathrm{H} 1$ and $2 c(2 / 3,1 / 3,1 / 4)$ in structure H2. The presence of either structure depends sensitively on the specific conditions used during sample synthesis (such as annealing environment and rate of cooling). Our refinement parameters indicate that this structural transition is especially sensitive to the Na content $x$, with $x \simeq 0.75$ as the critical concentration separating the two phases. By raising the temperature to around $T \simeq 323 \mathrm{~K}$, the high-symmetry structure $(\mathrm{H} 2)$ can be converted to the lower-symmetry structure (H1). We find that this structural transition also has signatures in measurements of the bulk magnetic and transport properties.

\section{Acknowledgments}

The work at MIT was supported primarily by the MRSEC Program of the National Science Foundation under grant number DMR 02-13282. Identification of commercial equipment in the text is not intended to imply recommendation or endorsement by the National Institute of Standards and Technology.
1 K. Takada, N. Sakurai, E. Takayama-Muromachi, F. Izumi, R. A. Dilanlan, and T. Sasaki, Nature 422 (2003).

2 R. Ray, A. Ghoshray, K. Ghoshray, and S. Nakamura, Phys. Rev. B 59, 9454 (1999).

${ }^{3}$ F. C. Chou, J. H. Cho, and Y. S. Lee (2004), condmat/0404061.

${ }^{4}$ M. L. Foo, Y. Wang, S. Watauchi, H. W. Zandbergen, T. He, R. J. Cava, and N. P. Ong (2003), cond- mat/0312174.

${ }^{5}$ H. Zandbergen, M. Foo, Q. Xu, V. Kumar, and R. J. Cava (2004), cond-mat/0403206.

${ }^{6}$ Q. Huang, M. Foo, J. Lynn, H. Zandbergen, G. Lawes, Y. Wang, B. H. Toby, A. Ramirez, N. Ong, and R. Cava (2004), cond-mat/0402255.

7 B. C. Sales, R. Jin, K. A. Affholter, P. Khalifah, G. M. Veith, and D. Mandrus (2004), cond-mat/0402379.

8 F. C. Chou, J. H. Cho, P. A. Lee, E. T. Abel, K. Matan, 
and Y. S. Lee, Phys. Rev. Lett. 92 (2004).

9 A. Larson and R. Von Dreele, Los Alamos National Laboratory, Internal Report (1994).

10 J. W. Lynn, Q. Huang, C. M. Brown, V. L. Miller, M. L. Foo, R. E. Schaak, C. Y. Jones, E. A. Mackey, and R. J. Cava, Physical Review B 68 (2003).
11 J. D. Jorgensen, M. Avdeev, D. G. Hinks, J. C. Burley, and S. Short, Physical Review B 68 (2003).

12 Q. Huang, M. Foo, J. Lynn, B. Toby, R. A. Pascal Jr., H. Zandbergen, and R. Cava (2004), unpublished. 